\title{
Exact Solutions and Conservation Laws of the Bogoyavlenskii Equation
}

\author{
M. INC ${ }^{a *}$, M.S. HASHEMI ${ }^{b}$ AND A. ISA AliYU ${ }^{a}$ \\ ${ }^{a}$ Department of Applied Mathematics, Firat University Turkey, 23119 Elazig, Turkey \\ ${ }^{b}$ Department of Mathematics, Basic Science Faculty, University of Bonab, Bonab 55517, Iran
}

(Received October 26, 2016; in final form January 4, 2018)

\begin{abstract}
In this paper, the Lie symmetry analysis method is performed for a Bogoyavlenskii equation. The symmetries and exact invariant solutions for the equation are retrieved for the first time. The conservation laws of the Bogoyavlenskii equation are constructed using the conservation laws theorem introduced by Ibragimov.
\end{abstract}

DOI: 10.12693 /APhysPolA.133.1133

PACS/topics: 02.30.Ik, 02.30.Jr, 47.35.Fg

\section{Introduction}

Mathematical modelling of scientific events is illustrated by nonlinear evolution equations (NLEEs). As such, it is important to obtain the general solutions of these models. The solutions of nonlinear partial differential equations (NLPDE's) provide a lot of information related to the character and structure of nonlinear models to researchers [1]. Many efficient techniques have been enhanced to provide useful information for researchers in the field of mathematics, physics and engineering. During the last decades, a variety of techniques have been recommended to analyze the behavior of NLPDEs [2-34].

Lie's classical theory is a basic of some generalizations. One of these generalizations is presented as a nonclassical approach by Bluman and Cole [3]. The most convenient description of Lie's invariance for initial value problems (IVPs) is given and summarized by Bluman et al. $[4,5]$. Details of the Lie symmetry technique is given in [6]. A technique for investigating the special symmetries of NLPDEs can also be found in [7].

In this paper, we will consider the Bogoyavlenskii (BK) equation given by [9-11]:

$$
\left\{\begin{array}{l}
a u_{t}+u_{x x y}+b u^{2} u_{y}+C u_{x} v=0 \\
u u_{y}-v_{x}=0
\end{array}\right.
$$

where $u$ is a shock wave, $v$ is the potential in physical process. The BK equation is a member of non-iso spectral scattering problems and breaking soliton theory [8]. Many authors have studied the BK equation and have reported the solutions of the equation. In Ref. [9], travelling wave and the singular manifold methods were used to retrieve the solitary wave solutions of the equation. The $G^{\prime} / G$ method was utilized to study the BK equation [10]. Also, the improved tanh-coth method was applied to study the solitary wave structure of the BK equation in [11].

*corresponding author; e-mail: minc@firat.edu.tr
In this work, we aim to investigate the solutions of the equation from the Lie symmetry point $[6,12,13]$ of view and then apply the new conservation theorem introduced by Ibragimov $[14,15]$ to establish the CLs for the BK equation.

\section{Lie symmetry analysis and exact invariant solutions}

In this section, we will extract the point symmetries $[6,11]$ of BK equation Eq. (1). The group invariant analysis and exact solutions will be investigated. Let us consider a one parameter Lie group of transformations

$$
\begin{aligned}
& t \rightarrow t+\epsilon \xi^{1}(t, x, y, u, v), \quad x \rightarrow x+\epsilon \xi^{2}(t, x, y, u, v), \\
& y \rightarrow y+\epsilon \xi^{3}(t, x, y, u, v), \quad u \rightarrow u+\epsilon \eta^{1}(t, x, y, u, v), \\
& v \rightarrow v+\epsilon \eta^{2}(t, x, y, u, v),
\end{aligned}
$$

with a small parameter $\epsilon \ll 1$. The vector field associated with the above group of transformations can be written as

$$
\Gamma=\xi^{1} \frac{\partial}{\partial x}+\xi^{2} \frac{\partial}{\partial t}+\xi^{3} \frac{\partial}{\partial y}+\eta^{1} \frac{\partial}{\partial u}+\eta^{2} \frac{\partial}{\partial v}
$$

where $\xi^{1}(t, x, y, u, v), \quad \xi^{2}(t, x, y, u, v), \quad \xi^{3}(t, x, y, u, v)$, $\eta^{1}(t, x, y, u, v), \eta^{2}(t, x, y, u, v)$ are the infinitesimals to be determined. For the system Eq. (1), the prolongation of $\Gamma$ is given by $P_{r}^{3}$. Thus, the invariance condition [12] can be written as

$$
\left.P_{r}^{3} \Gamma\left(\Delta_{1}\right)\right|_{\Delta_{1}=0}=0,\left.\quad P_{r}^{1} \Gamma\left(\Delta_{2}\right)\right|_{\Delta_{2}=0}=0,
$$

where

$$
\left\{\begin{array}{l}
\Delta_{1}=a u_{t}+u_{x x y}+b u^{2} u_{y}+C u_{x} v=0, \\
\Delta_{2}=u u_{y}-v_{x}=0 .
\end{array}\right.
$$

Application of the prolongations of Eq. (4) to Eq. (1) results in a huge overdetermined system of linear partial differential equations (LPDEs) for the infinitesimals. The solutions of the system of LPDEs lead us to some cases split based on the constants [12]:

Case 1: $a \neq 0, b=C=0$. In this case, by using the determining equations, we obtain

$$
\xi^{1}(t, x, y, u, v)=C_{4}+t C_{5},
$$




$$
\begin{aligned}
& \xi^{2}(t, x, y, u, v)=C_{1}-\frac{x C_{2}}{2}, \\
& \xi^{2}(t, x, y, u, v)=y\left(C_{2}+C_{5}\right)+C_{6}, \\
& \eta^{1}(t, x, y, u, v)=u\left(-\frac{C_{2}}{4}+C_{3}\right), \\
& \eta^{2}(t, x, y, u, v)=-v\left(2 C_{2}-2 C_{3}+C_{5}\right)+F_{1}(t, v),
\end{aligned}
$$

where $C_{i}(i=1, \ldots, 5)$ are constants. Hence, the related vector field becomes

$$
\begin{aligned}
& \Gamma_{1}=\frac{\partial}{\partial y}, \quad \Gamma_{2}=\frac{\partial}{\partial x}, \quad \Gamma_{3}=\frac{\partial}{\partial t}, \quad \Gamma_{4}=F_{1}(t, y) \frac{\partial}{\partial v} \\
& \Gamma_{5}=u \frac{\partial}{\partial u}+2 v \frac{\partial}{\partial v}, \quad \Gamma_{6}=t \frac{\partial}{\partial u}-v \frac{\partial}{\partial v}+y \frac{\partial}{\partial y} \\
& \Gamma_{7}=-\frac{\partial}{\partial u}-8 v \frac{\partial}{\partial v}-\frac{\partial}{\partial x}+4 y \frac{\partial}{\partial y} .
\end{aligned}
$$

For the generator $\Gamma_{1}+\Gamma_{2}+\Gamma_{3}+\Gamma_{5}=\frac{\partial}{\partial y}+\frac{\partial}{\partial x}+\frac{\partial}{\partial t}+$ $u \frac{\partial}{\partial u}+2 v \frac{\partial}{\partial v}$, the corresponding similarity variables are

$$
\left\{\begin{array}{l}
u(t, x, y)=\mathrm{e}^{t} F_{1}\left(\xi_{1}, \xi_{2}\right), \\
v(t, x, y)=\mathrm{e}^{2 t} F_{2}\left(\xi_{1}, \xi_{2}\right),
\end{array}\right.
$$

where

$$
\xi_{1}=x-t, \quad \xi_{2}=y-t .
$$

Substituting Eq. (1) into the BK equation, we obtain the following system of ordinary differential equations (ODEs):

$$
\left\{\begin{array}{l}
b \mathrm{e}^{3 t} F_{1} \frac{\partial F_{1}}{\partial \xi_{2}}+a\left(\mathrm{e}^{t} F_{1}+\mathrm{e}^{t}\left(-\frac{\partial F_{1}}{\partial \xi_{2}}-\frac{\partial F_{1}}{\partial \xi_{1}}\right)\right) \\
\quad+c \mathrm{e}^{3 t} F_{2} \frac{\partial F_{1}}{\partial \xi_{1}}+\mathrm{e}^{t} \frac{\partial^{3} F_{1}}{\partial \xi_{1}^{2} \xi_{2}}=0, \\
F_{1} \frac{\partial F_{1}}{\partial \xi_{2}}-\frac{\partial F_{2}}{\partial \xi_{1}}=0 .
\end{array}\right.
$$

Setting $b=C=0$ in Eq. (10), we acquire

$$
\left\{\begin{aligned}
a F_{1}-a \frac{\partial F_{1}}{\partial \xi_{1}}-a \frac{\partial F_{1}}{\partial \xi_{2}}+\frac{\partial^{3} F_{1}}{\partial \xi_{1}^{2} \xi_{2}} & =0, \\
F_{1} \frac{\partial F_{1}}{\partial \xi_{2}}-\frac{\partial F_{2}}{\partial \xi_{1}} & =0 .
\end{aligned}\right.
$$

Solving Eq. (11), we get the following exponential function solution:

$$
\begin{aligned}
F_{1}\left(\xi_{1}, \xi_{2}\right) & =C_{1} \exp \left(\frac{2 C_{3}^{2} \xi_{2}+a \xi_{1}+\xi_{1} \psi}{2 C_{3}}\right) \\
+C_{2} \exp & \left(\frac{2 C_{3}^{2} \xi_{2}+a \xi_{1}-\xi_{1} \psi}{2 C_{3}}\right), \\
F_{2}\left(\xi_{1}, \xi_{2}\right) & =\frac{2 C_{1} C_{2}}{a} \exp \left(\frac{2 C_{3}^{2} \xi_{2}+a \xi_{1}}{C_{3}}\right) \\
+\frac{C_{1}^{2}}{a+\psi} & \exp \left(\frac{2 C_{3}^{2} \xi_{2}+a \xi_{1}+\xi_{1} \psi}{C_{3}}\right) \\
+\frac{C_{2}^{2}}{a-\psi} & \exp \left(\frac{2 C_{3}^{2} \xi_{2}+a \xi_{1}-\xi_{1} \psi}{C_{3}}\right)+\Gamma\left(\xi_{2}\right) .
\end{aligned}
$$

Subsequently, we obtain the following solution for the BK equation:

$$
\begin{gathered}
u(t, x, y)=\left\{C_{1} \exp \left(\frac{2 C_{3}^{2} \xi_{2}+a \xi_{1}+\xi_{1} \psi}{2 C_{3}}\right)\right. \\
\left.+C_{2} \exp \left(\frac{2 C_{3}^{2} \xi_{2}+a \xi_{1}-\xi_{1} \psi}{2 C_{3}}\right)\right\} \mathrm{e}^{t},
\end{gathered}
$$

$$
\begin{aligned}
& v(t, x, y)=\left\{\frac{2 C_{1} C_{2}}{a} \exp \left(\frac{2 C_{3}^{2} \xi_{2}+a \xi_{1}}{C_{3}}\right)+\right. \\
& \frac{C_{1}^{2}}{a+\psi} \exp \left(\frac{2 C_{3}^{2} \xi_{2}+a \xi_{1}+\xi_{1} \psi}{C_{3}}\right)+ \\
& \left.\frac{C_{2}^{2}}{a-\psi} \exp \left(\frac{2 C_{3}^{2} \xi_{2}+a \xi_{1}-\xi_{1} \psi}{C_{3}}\right)+\Gamma\left(\xi_{2}\right)\right\} \mathrm{e}^{2 t},
\end{aligned}
$$

where $\psi=\sqrt{a\left(a+4 C_{3}^{2}-4 C_{3}\right)}$ and $\xi_{1}, \xi_{2}$ are given in Eq. (9)

Case 2: $a \neq 0, C \neq 0$. The coefficient functions of vector field in this case are

$$
\begin{aligned}
& \xi^{1}(t, x, y, u, v)=C_{2}+t C_{3}, \\
& \xi^{2}(t, x, y, u, v)=x C_{1}+F_{1}(t), \\
& \xi^{3}(t, x, y, u, v)=y\left(-2 C_{1}+C_{3}\right)+C_{4}, \\
& \eta^{1}(t, x, y, u, v)=-u C_{1}, \\
& \eta^{2}(t, x, y, u, v)=-v\left(C_{1}-C_{3}\right)+\frac{a F_{1}^{\prime}}{C},
\end{aligned}
$$

where $C_{i}(i=1, \ldots, 4)$ are constants. Hence the related vector

$$
\begin{aligned}
\Gamma_{1} & =\frac{\partial}{\partial y}, \quad \Gamma_{2}=\frac{\partial}{\partial t}, \quad \Gamma_{3}=t \frac{\partial}{\partial t}-v \frac{\partial}{\partial v}+y \frac{\partial}{\partial y^{\prime}}, \\
\Gamma_{4} & =\frac{a F_{1}^{\prime}}{C} \frac{\partial}{\partial v}+F_{1}(t) \frac{\partial}{\partial x} \\
\Gamma_{5} & =-u \frac{\partial}{\partial u}+v \frac{\partial}{\partial v}+x \frac{\partial}{\partial x}-2 y \frac{\partial}{\partial y} .
\end{aligned}
$$

The similarity variables for the following vector field:

are

$$
\Gamma_{2}+\Gamma_{5}=\frac{\partial}{\partial t}+x \frac{\partial}{\partial x}-2 y \frac{\partial}{\partial y}-u \frac{\partial}{\partial u}+v \frac{\partial}{\partial v}
$$

$$
\left\{\begin{array}{l}
u(t, x, y)=\mathrm{e}^{-t} F_{2}\left(\xi_{1}, \xi_{2}\right), \\
v(t, x, y)=\mathrm{e}^{t} F_{1}\left(\xi_{1}, \xi_{2}\right),
\end{array}\right.
$$

where

$$
\xi_{1}=x-t, \quad \xi_{2}=y-t,
$$

satisfying the ODE

$$
\begin{aligned}
& b F_{2}^{2} \frac{\partial F_{2}}{\partial \xi_{2}}+C F_{1} \frac{\partial F_{2}}{\partial \xi_{1}}-a F_{2}+\frac{\partial^{3} F_{1}}{\partial \xi_{1}^{2} \xi_{2}}=0, \\
& F_{1} \frac{\partial F_{2}}{\partial \xi_{2}}-\frac{\partial F_{1}}{\partial \xi_{1}}=0 \rightarrow \frac{\partial F_{1}}{\partial \xi_{1}} \rightarrow F_{1}\left(\xi_{1}, \xi_{2}\right)=G\left(\xi_{2}\right), \\
& \frac{\partial F_{2}}{\partial \xi_{2}}=0, \frac{\partial F_{2}}{\partial \xi_{1}} \rightarrow F_{2}\left(\xi_{1}, \xi_{2}\right)=C_{1} .
\end{aligned}
$$

$G\left(\xi_{2}\right)$ is an arbitrary function. Thus, the exact solution for the BK equation for this case is given by

$$
\left\{\begin{array}{l}
u(t, x, y)=e^{-t} C_{1}, \\
v(t, x, y)=e^{t} G(y-t) .
\end{array}\right.
$$

Case 3: $a \neq 0, b \neq 0 C=0$. For this case, the coefficient functions of vector field are

$$
\begin{aligned}
& \xi^{1}(t, x, y, u, v)=C_{3}+t C_{4}, \\
& \xi^{2}(t, x, y, u, v)=C_{1}-\frac{2 x C_{2}}{3},
\end{aligned}
$$




$$
\begin{aligned}
& \xi^{3}(t, x, y, u, v)=y\left(\frac{4 C_{3}}{3}+C_{4}\right)+C_{5}, \\
& \eta^{1}(t, x, y, u, v)=\frac{2 u C_{2}}{3}, \\
& \eta^{2}(t, x, y, u, v)=-v\left(\frac{2 C_{2}}{3}+C_{4}\right)+F_{1}(t, y),
\end{aligned}
$$

where $C_{i}(i=1, \ldots, 5)$ are constants. Hence the related vector fields for this case are given by

$$
\begin{aligned}
& \Gamma_{1}=\frac{\partial}{\partial y}, \quad \Gamma_{2}=\frac{\partial}{\partial x}, \quad \Gamma_{3}=\frac{\partial}{\partial t}, \\
& \Gamma_{4}=F_{1}(t, y) \frac{\partial}{\partial v^{\prime}}, \quad \Gamma_{5}=t \frac{\partial}{\partial t}-v \frac{\partial}{\partial v}+y \frac{\partial}{\partial y}, \\
& \Gamma_{6}=u \frac{\partial}{\partial u}-v \frac{\partial}{\partial v}+2 y \frac{\partial}{\partial y}-x \frac{\partial}{\partial x} .
\end{aligned}
$$

For the generator $\Gamma_{1}+\Gamma_{2}+\Gamma_{3}+\Gamma_{4}=\frac{\partial}{\partial t}+\frac{\partial}{\partial x}+\frac{\partial}{\partial y}+\frac{\partial}{\partial v}$, and choosing the function $F_{1}(t, y)=1$, Eq. (1) transforms to the following ODE:

$$
\left\{\begin{array}{l}
u(t, x, y)=F_{1}\left(\xi_{1}, \xi_{2}\right), \\
v(t, x, y)=t+F_{2}\left(\xi_{1}, \xi_{2}\right),
\end{array}\right.
$$

where

$$
\xi_{1}=x-t, \quad \xi_{2}=y-t,
$$

satisfying the following ODE:

$$
\begin{aligned}
& b F_{2}^{2} \frac{\partial F_{1}}{\partial \xi_{2}}-a \frac{\partial F_{1}}{\partial \xi_{2}}-a \frac{\partial F_{1}}{\partial \xi_{1}}+\frac{\partial^{3} F_{1}}{\partial \xi_{1}^{2} \xi_{2}}=0, \\
& F_{1} \frac{\partial F_{1}}{\partial \xi_{2}}-\frac{\partial F_{2}}{\partial \xi_{1}}=0 .
\end{aligned}
$$

Solving the above system of ODE, we obtain

$$
\begin{aligned}
& F_{1}\left(\xi_{1}, \xi_{2}\right)=\frac{\sqrt{-3 a b\left(a+\psi-4 C_{2}^{2}\right)}}{2 C_{2} b} \\
& \quad \times \tanh \left(C_{1}-\frac{(a+\psi) \xi_{1}}{4 C_{2}}+C_{2} \xi_{2}\right), \\
& F_{2}\left(\xi_{1}, \xi_{2}\right)=\frac{3 a\left(4 C_{2}^{2}-a-\psi\right)}{2 b(a+\psi)} \\
& \quad \times \operatorname{sech}^{2}\left(\frac{-4 C_{1} C_{2}+\xi_{1} a+\xi_{1} \psi-4 C_{2}^{2} \xi_{2}}{4 C_{2}}\right) \\
& \quad+G\left(\xi_{2}\right) .
\end{aligned}
$$

Subsequently, we obtain the following topological and non-topological soliton solutions for the BK equation:

$$
\begin{aligned}
& u(t, x, y)=\frac{\sqrt{-3 a b\left(a+\psi-4 C_{2}^{2}\right)}}{2 C_{2} b} \\
& \quad \times \tanh \left(C_{1}-\frac{(a+\psi) \xi_{1}}{4 C_{2}}+C_{2} \xi_{2}\right), \\
& v(t, x, y)=t+\frac{3 a\left(4 C_{2}^{2}-a-\psi\right)}{2 b(a+\psi)} \\
& \quad \times \operatorname{sech}^{2}\left(\frac{-4 C_{1} C_{2}+\xi_{1} a+\xi_{1} \psi-4 C_{2}^{2} \xi_{2}}{4 C_{2}}\right) \\
& \quad+G\left(\xi_{2},\right.
\end{aligned}
$$

where $\psi=\sqrt{a^{2}-8 C_{2}^{2} a}$ and $\xi_{1}, \xi_{2}$ are given in Eq. (24).

\section{Non-local conservation laws}

In this section, we first give a brief introduction of the new conservation theorem [14] and then we apply the method to construct the conservation laws of the BK equation Eq. (1). We first consider the following Theorem from [15]:

Theorem 3.1 The system of $\bar{m}$ differential equations

$$
F_{\alpha}\left(\bar{x}, u, u_{1}, \ldots, u_{s}\right)=0, \quad \alpha=1, \ldots, \bar{m},
$$

having $m$ dependent variables $u=\left(u^{1}, \ldots, u^{m}\right)$ has adjoint equation

$$
\begin{aligned}
& F_{\alpha}^{*}\left(\bar{x}, u, z, u_{1}, z_{1}, \ldots, u_{s}, z_{s}\right)=\frac{\delta\left(z^{\bar{\beta}} F_{\bar{\beta}}\right)}{\delta u^{\alpha}}, \\
& \alpha=1, \ldots, m,
\end{aligned}
$$

and Lagrangian given by

$$
\mathcal{L}=z^{\bar{\beta}} F_{\bar{\beta}},
$$

where $z^{\bar{\beta}}=z^{\bar{\beta}}(\bar{x}, t)$ is a nonlocal dependent variable.

To investigate the CLs of Eq. (1), we let

$$
\left\{\begin{array}{l}
F_{1}=a u_{t}+u_{x x y}+b u^{2} u_{y}+c u_{x} v=0, \\
F_{2}=u u_{y}-v_{x}=0 .
\end{array}\right.
$$

We compute the formal Lagrangian using Eq. (30):

$\mathcal{L}=w\left(-u u_{y}+v_{t}\right)+z\left(b u^{2} u_{y}+u_{t}+C v u_{x}+u_{x x y}\right)$,

where $z=z(t, x, y)$ and $w=w(t, x, y)$ are new dependent variables called the nonlocal variables. The adjoint system can be obtained using Eq. (29) as

$$
F_{1}^{*}=\frac{\delta \mathcal{L}}{\delta u}=0, \quad F_{2}^{*}=\frac{\delta \mathcal{L}}{\delta v}=0 .
$$

After the above operation, the adjoint becomes

$$
\left\{\begin{array}{l}
F_{1}^{*}=a u_{t}+u_{x x y}+b u^{2} u_{y}+c u_{x} v, \\
F_{2}^{*}=u u_{y}-v_{x} .
\end{array}\right.
$$

In the following we recall the following theorem given by Ibragimov [14].

\section{Theorem 3.2 Let}

$$
\Gamma=\xi_{i}\left(\bar{x}, u, u_{1}, \ldots\right) \frac{\partial}{\partial \bar{x}^{i}}+\eta_{\alpha}\left(\bar{x}, u, u_{1}, \ldots\right) \frac{\partial}{\partial u^{\alpha}},
$$

be a symmetry (point, Bäcklund or contact) of Eq. (28) with an adjoint Eq. (29) and a Lagrangian Eq. (30). Then Eq. (28) satisfies the conservation equation

$$
\left.D_{i}\left(T^{i}\right)\right|_{(E q .28=0)}=0,
$$

with

$$
\begin{aligned}
& T^{i}=\xi_{i} \mathcal{L}+W^{\alpha}\left[\frac{\partial \mathcal{L}}{\partial u_{i}^{\alpha}}-D_{j}\left(\frac{\partial \mathcal{L}}{\partial u_{i j}^{\alpha}}\right)+D_{j} D_{k} \frac{\partial \mathcal{L}}{\partial u_{i j k}^{\alpha}} \ldots\right] \\
& +D_{j}\left(W^{\alpha}\right)\left[\frac{\partial \mathcal{L}}{\partial u_{i j}^{\alpha}}-D_{k}\left(\frac{\partial \mathcal{L}}{\partial u_{i j k}^{\alpha}}\right)+\ldots\right] \\
& +D_{j} D_{k}\left(W^{\alpha}\right)\left[\frac{\partial \mathcal{L}}{\partial u_{i j k}^{\alpha}}\right]+\ldots,
\end{aligned}
$$

and

$$
W^{\alpha}=\eta_{\alpha}-\xi_{j} u_{j}^{\alpha} .
$$

The components $\left(T_{i}^{x}, T_{i}^{y}, T_{i}^{t}\right)(i=1,2, \ldots, 9)$ are called conserved vectors. Using the symmetries, the Lagrangian 
Eq. (32) and the conservation formulae Eq. (37), we obtain the following set of conserved quantities for each of the point symmetries. In constructing the CLs for the vector fields admitted by the BK equation for the three cases discussed in Sect. 2, we extracted nine vector fields without repetition.

- We first consider the vector field $\Gamma_{1}=\frac{\partial}{\partial y}$, it admits the following conserved vectors:

$$
\begin{aligned}
& \boldsymbol{T}_{1}^{x}=\frac{-a w v_{y}+u_{y y} z_{x}+z_{y} u_{x y}-u_{y}\left(C z v+z_{x y}\right)-z u_{x y y}}{a} \\
& \boldsymbol{T}_{1}^{y}=\frac{z_{x} u_{x y}+u_{y}\left(a u w-b u^{2} z-z_{x x}\right)-z u_{x x y}}{a} \\
& \boldsymbol{T}_{1}^{t}=-z u_{y} .
\end{aligned}
$$

- Lie point symmetry generator $\Gamma_{2}=\frac{\partial}{\partial x}$ yields the conserved vector with components

$$
\begin{aligned}
& \boldsymbol{T}_{2}^{x}=\frac{-a u w u_{y}+z_{x} u_{x y}-u_{x}\left(C z v+z_{x y}\right)+z_{y} u_{x x}-z u_{x x y}}{a}, \\
& \boldsymbol{T}_{2}^{y}=\frac{z_{x} u_{x x}-u_{x}\left(-a u w+b u^{2} z+z_{x x}\right)-z u_{x x x}}{a}, \\
& \boldsymbol{T}_{2}^{t}=-z u_{x} .
\end{aligned}
$$

- The Lie point symmetry generator $\Gamma_{3}=\frac{\partial}{\partial t}$, yield the conserved quantities

$$
\begin{aligned}
& \boldsymbol{T}_{3}^{x}=\frac{-a w v_{t}+u_{t y} z_{x}+z_{y} u_{x t}-u_{t} z_{x y}-z\left(C v u_{t}+u_{x y t}\right)}{a}, \\
& \boldsymbol{T}_{3}^{y}=\frac{z_{x} u_{t x}-u_{t}\left(-a u w+b u^{2} z+z_{x x}\right)-z u_{x x t}}{a}, \\
& \boldsymbol{T}_{3}^{y}=\frac{-a u w u_{y}+b u^{2} z u_{y}+a w v_{x}+z\left(C v u_{x}+u_{x x y}\right)}{a} .
\end{aligned}
$$

- Lie point symmetry generator $\Gamma_{4}=$ $F(y, t) \frac{\partial}{\partial v}$ yields the conserved vector with components

$$
\begin{aligned}
& \boldsymbol{T}_{4}^{x}=F(y, t) w, \\
& \boldsymbol{T}_{4}^{y}=0, \\
& \boldsymbol{T}_{4}^{t}=0 .
\end{aligned}
$$

- Lie point symmetry generator $\Gamma_{5}=2 v \frac{\partial}{\partial v}+$ $u \frac{\partial}{\partial u}$ yields the conserved vector with components

$$
\begin{aligned}
& \boldsymbol{T}_{5}^{x}=\frac{2 a w v-z_{y} u_{x}-u_{y} z_{x}+z u_{x y}+u\left(C z v+z_{x y}\right)}{a}, \\
& \boldsymbol{T}_{5}^{y}=\frac{-a u^{2} w+b u^{3} z-u_{x} z_{x}+z u_{x x}+u z_{x x}}{a}, \\
& \boldsymbol{T}_{5}^{t}=u z .
\end{aligned}
$$

- Lie point symmetry generator $\Gamma_{6}=$ $2 y \frac{\partial}{\partial y}+u \frac{\partial}{\partial u}-x \frac{\partial}{\partial x}-v \frac{\partial}{\partial v}$ yields the conserved vector with components

$$
\begin{aligned}
& \boldsymbol{T}_{6}^{x}=a^{-1}\left(-2 C y z v u_{y}-a w\left(v-x u u_{y}\right.\right. \\
& \left.\quad+2 y v_{y}\right)+C x z v u_{x}-2 z_{y} u_{x}+u_{y} z_{x}+2 y u_{y y} z_{x} \\
& \quad+2 y z_{y} u_{x y}-x z_{x} u_{x y}-2 y u_{y} z_{x y}+x u_{x} z_{x y} \\
& \quad+u\left(C z v+z_{x y}\right)-2 y z u_{x y y}-x z_{y} u_{x x} \\
& \left.\quad+x z u_{x x y}\right) \\
& \boldsymbol{T}_{6}^{y}=a^{-1}\left(b u^{3}+u^{2}\left(-a w+b z\left(-2 y u_{y}\right.\right.\right. \\
& \left.\left.\quad+x u_{x}\right)\right)+2 y z_{x} u_{x y}+3 z u_{x x}-x z_{x} u_{x x} \\
& \quad-2 y u_{y} z_{x x}+u\left(a w\left(2 y u_{y}-x u_{x}\right)+z_{x x}\right) \\
& \left.\quad+u_{x}\left(-2 z_{x}+x z_{x x}\right)-2 y z u_{x x y}+x z u_{x x x}\right), \\
& \boldsymbol{T}_{6}^{t}=z\left(u-2 y u_{y}+x u_{x}\right) .
\end{aligned}
$$

- Lie point symmetry generator $\Gamma_{7}=t \frac{\partial}{\partial t}+y \frac{\partial}{\partial y}-v \frac{\partial}{\partial y}$ yields the conserved vector with components

$$
\begin{aligned}
\boldsymbol{T}_{7}^{x} & =a^{-2}\left(-w\left(v+t v_{t}+y v_{y}\right)+(C z v\right. \\
& \left.+z_{x y}\right)\left(\left(-a y+b t u^{2}\right) u_{y}+t\left(C v u_{x}+u_{x x y}\right)\right) \\
& +z_{x}\left(a u_{y}-2 b t u u_{y}^{2}+\left(a y-b t u^{2}\right) u_{y y}\right. \\
& \left.-t\left(C v_{y} u_{x}+C v u_{x y}+u_{x x y y}\right)\right) \\
& -z_{y}\left(-a y u_{x y}+t\left(2 b u u_{x} u_{y}+C u_{x} v_{x}\right.\right. \\
& \left.\left.+b u^{2} u_{x y}+C v u_{x x}+u_{x x x y}\right)\right)+z\left(-a\left(u_{x y}\right.\right. \\
& \left.+y u_{x y y}\right)+t\left(2 b u_{y}^{2} u_{x}+2 b u u_{y y} u_{x}-4 b u u_{y} u_{x y}\right. \\
& +b u^{2} u_{x y y}+C\left(v_{x} u_{x y}+u_{x} v_{x y}+v_{y} u_{x x}\right. \\
& \left.\left.\left.\left.+v u_{x x y}\right)+u_{x x x y y}\right)\right)\right) \\
\boldsymbol{T}_{7}^{y} & =a^{-2}\left(-a b t u^{3} w u_{y}+b^{2} t u^{4} z u_{y}\right. \\
& -C t u_{x} z_{x} v_{x}+a y z_{x} u_{x y}-C t v z_{x} u_{x x} \\
& -a y u_{y} z_{x x}+C t v u_{x} z_{x x}+t z_{x x} u_{x x y} \\
& +b u^{2}\left(-t z_{x} u_{x y}+t u_{y} z_{x x}+z\left(C t v u_{x}\right.\right. \\
& \left.\left.+2 t u_{x x y}\right)\right)+u\left(2 b t \left(u_{x}\left(-u_{y} z_{x}+2 z u_{x y}\right)\right.\right. \\
& \left.\left.+z u_{y} u_{x x}\right)+a w\left(a y u_{y}-t\left(C v u_{x}+u_{x x y}\right)\right)\right) \\
& -t z_{x} u_{x x x y}+z^{2}\left(\left(-a y+b t u^{2}\right) u_{y}+t\left(C v u_{x}\right.\right. \\
& \left.\left.\left.+u_{x x y}\right)\right)\right) \\
\boldsymbol{T}_{7}^{t} & =z\left(\left(-a y+b t u^{2}\right) u_{y}+t\left(C v u_{x}+u_{x x y}\right)\right) .
\end{aligned}
$$

- Lie point symmetry generator $\Gamma_{8}=$ $-2 x \frac{\partial}{\partial x}+4 y \frac{\partial}{\partial y}-8 v \frac{\partial}{\partial v}-u \frac{\partial}{\partial u}$ yields the conserved vector with components 


$$
\begin{aligned}
& \boldsymbol{T}_{8}^{x}=-a^{-1}\left(4 C y z v u_{y}+2 a w\left(4 v-x u u_{y}\right.\right. \\
& \left.\quad+2 y v_{y}\right)-2 C x z v u_{x}+z_{y} u_{x}-5 u_{y} z_{x} \\
& \quad-4 y u_{y y} z_{x}+3 y z_{y} u_{x y}+2 x z_{x} u_{x y}+4 y u_{y} z_{x y} \\
& \quad-2 x u_{x} z_{x y}+u\left(C z v+z_{x y}\right)+4 y z u_{x y y} \\
& \left.\quad+2 x z_{y} u_{x x}-2 x z u_{x x y}\right) . \\
& \boldsymbol{T}_{8}^{y}=-b u^{3} z+u^{2}\left(a w+2 b z\left(-2 y u_{y}+x u_{x}\right)\right) \\
& \quad+4 y z_{x} u_{x y}+3 z u_{x x}-2 x z_{x} u_{x x}+u\left(w \left(4 a y u_{y}\right.\right. \\
& \left.\left.\quad-2 a x u_{x}\right)-z_{x x}\right)-4 y u_{y} z_{x x}-u_{x}\left(z_{x}\right. \\
& \left.\quad-2 x z_{x x}\right)-4 y z u_{x x y}+2 x z u_{x x x}, \\
& \boldsymbol{T}_{8}^{t}=-z\left(u+4 y u_{y}-2 x u_{x}\right) .
\end{aligned}
$$

- Finally, the Lie point symmetry generator $\Gamma_{9}=\frac{a F_{1}^{\prime}(t)}{c} \frac{\partial}{\partial v}+F_{1}(t) \frac{\partial}{\partial x}$ yields the conserved vector with components

$$
\begin{aligned}
\boldsymbol{T}_{9}^{x} & =\frac{1}{a C}\left(a w\left(a \mathcal{F}_{1}^{\prime}-C u \mathcal{F}_{1}(t) u_{y}\right)\right. \\
& -C \mathcal{F}_{1}(t)\left(-z_{x} u_{x y}+u_{x}\left(C z v+z_{x y}\right)\right. \\
& \left.\left.-z_{y} u_{x x}+z u_{x x y}\right)\right) \\
\boldsymbol{T}_{9}^{y} & =\frac{\mathcal{F}_{1}(t)\left(z_{x} u_{x x}-u_{x}\left(-a u w+b u^{2} z+z_{x x}\right)-z u_{x x x}\right)}{a}, \\
\boldsymbol{T}_{9}^{t} & =-z \mathcal{F}_{1}(t) u_{x} .
\end{aligned}
$$

\section{Conclusion}

We have used Lie symmetry methods to find the exact solutions of the BK equation. The obtained solutions are expressed in terms of trigonometric, algebraic and exponential functions. In particular, nine sets of nontrivial CLs of the BK are constructed using the general CLs Theorem. All the obtained solutions satisfy the BK equation. This was verified using the Wolfram Mathematica 9 .

\section{References}

[1] M. Mirzazadeh, M. Eslami, A. Biswas, Optic 125, 6874 (2014).

[2] M.E. Zayed, K.A. Gepreel, M.M. El Horbaty, Appl. Anal. 87, 509 (2008).

[3] G.W. Bluman, J.D. Cole, J. Math. Mech. 18, 1025 (1969).

[4] G.W. Bluman, S. Kumei, Symmetries and Differential Equations, Springer, Berlin 1989.

[5] G.W. Bluman, S.C. Anco, Symmetry and Integration Methods for Differential Equations. Applied Mathematical Sciences, Springer, New York 2002.

[6] P.J. Olver, Application of Lie Groups to Differential Equations, Springer, Berlin 1986.
[7] C.A. Gomez, A. Salas, Appl. Math. Comp. 216, 3089 (2010).

[8] O.I. Bogoyavlenskii, Russ. Math. Surveys 12, 1 (1990).

[9] Y. Peng, M. Shen, Pramana J. Phys. 67, 3 (2006).

[10] A. Malik, F. Chand, H. Kumar, S.C. Misshra, Comp. Math. Appl. 64, 2850 (2012).

[11] C.A. Gomez, Appl. Math. Sci. 89, 4443 (2015).

[12] E. Buhe, G.W. Bluman, J. Math. Phys. 56, 101501 (2015).

[13] M.S. Hashemi, S. Abbasbandy, H.H. Alhuthali, M.M. Alsulam, Rom. J. Phys. 60, 904 (2015).

[14] N.H. Ibragimov, J. Math. Anal. Appl. 333, 311 (2007).

[15] N.H. Ibragimov, $A L G A$ 7/8 44, 1 (2011).

[16] Z. Fu, S. Liu, Q. Zhao, Phys. Lett. A 290, 72 (2001).

[17] W. Heremen, W. Zhuang, A MACSYMA Program for the Hirota Method, in: Proc. 13th World Congress on Computation and Applied Mathematics, Dublin (Ireland), 1991, Eds. R. Vichnevetsky, J.J.H. Miller, p. 842 .

[18] W. Heremen, W. Zhuang, Comput. Appl. Math. II Diff. Equat. 287, 349 (1992).

[19] W. Heremen, W. Zhuang, A MACSYMA Program for the Hirota Method, in Ref. [17], p. 22.

[20] E.M.E. Zayed, H.A. Zedan, K.A. Gepreel, Applic. Anal. 84, 523 (2005).

[21] Z.S. Feng, J. Phys. Appl. 35, 343 (2002).

[22] M. Eslami, M. Mirzadeh, Eur. Phys. J. Plus 128, 140 (2013).

[23] R.K. Raslam, Nonlin. Dyn. 53, 281 (2008).

[24] F. Tascan, Appl. Math. Comput. 207, 279 (2009).

[25] S. Abbasbandy, A. Shirzadi, Commun. Nonlinear Sci. Num. Simul. 15, 1759 (2000).

[26] H. Jafari, A. Sooraki, C.M. Khalique, Optik 124, 3929 (2013).

[27] N.H. Ibragimov, V.F. Kovalev, Aproximate and Renormgroup Symmetries, Beiging and Springer, Germany 2009.

[28] T.R. Ding, C.Z. Li, Ordinary Differential Equations, Peking University Press, Peking 1996.

[29] E.V. Krishnan, H. Trihi, M. Labidi, A. Biswas, Nonlin. Dyn. 66, 497 (2011).

[30] A. Biswas, A.H. Kara, Appl. Math. Comput. 217, 929 (2010).

[31] A. Biswas, A.H. Kara, L. Moraru, A.H. Bokhari, F.D. Zaman, Proc. Rom. Acad. A 15, 123 (2014).

[32] R. Morris, A.H. Kara, A. Biswas, Nonlin. Anal. Mod. Control. 18, 153 (2013).

[33] P. Razborova, A.H. Kara, A. Biswas, Nonlin. Dyn. 79, 743 (2015).

[34] E. Yasar, I.B. Girresunlu, Acta Phys. Pol. A 128, 252 (2015). 